\title{
More evidence required to establish link between premature birth and altered oral development
}

\author{
Does premature birth affect oral development?
}

\begin{abstract}
Paulsson L, Bondemark L, Soderfeldt B. A systematic review of the consequences of premature birth on palatal morphology, dental occlusion, tooth-crown dimensions, and tooth maturity and eruption. Angle Orthod 2004; 74:269-279
\end{abstract}

Data sources PubMed provided the primary data source with references from identified papers being reviewed to find additional studies.

Study selection Reports were selected of controlled studies that provided quantitative data on the effects of premature birth on jaws, dentition, dental physiology and tooth anomalies, which were published in the English language.

Data extraction and synthesis Two independent evaluators extracted data describing the year of publication, definition of prematurity, sample size, material and age, methods and measurements, outcomes and authors' conclusions. Correlation between deformity/alteration and prematurity, intubation and sucking habit were considered. Methodological soundness was assessed and the quality of each article categorised as low, medium or high.

Results A total of 13 articles met the inclusion criteria. Quality was judged to be high for three studies, medium for nine studies and low for a single study. The chosen studies described altered palatal morphology $(n=5)$, altered dental maturation $(n=5)$ and altered tooth-crown dimensions $(n=3)$. The limited evidence suggests there may be a correlation between prematurity and altered palate morphology in the short term, with oral intubation as a contributing factor. If corrected age was considered no delay was found in maturation.

Conclusions This systematic review revealed contradictory results and a dearth of longitudinal studies in this subject area. Further welldesigned controlled and longitudinal studies are required before any conclusions can be made regarding the consequences of premature birth on oral development.

Address for correspondence: Lars Bondemark, Department of Orthodontics, Faculty of Odontology, Malmö University, Malmö, Sweden. E-mail: lars.bondemark@od.mah.se

\section{Commentary}

As this article states, "The great improvement in neonatal health and intensive care in the last 20 years has led to increasing survival of premature infants". Thus, dentists and orthodontists are treating increasing numbers of children who were born prematurely. Unless we ask about this specifically when taking a medical history, have access to full hospital notes or are informed by the parents, we may be unaware of this important formative period in the child's early life. The only obvious clinical sign to many practitioners would be the occasional presence of visible enamel defects.

This review addresses the relationship between prematurity (and the use of oro- and nasogastric tubes) and altered palatal morphology, dental occlusion, tooth-crown dimensions and tooth maturation. All of these criteria are of interest to orthodontic practitioners. The first two may increase the need for orthodontics as well as the complexity of management. The second two effects could complicate or delay orthodontic treatment.

Every systematic review should define its aim: here, the clinical problem is laid out in a comprehensive introduction and the paper then sets out three distinct questions to be answered:

Does prematurity result in alterations of palatal morphology, dental occlusion, tooth-crown dimensions, and tooth maturation and eruption?

What role does oral intubation play in the appearance of the alterations?

Are the alterations in morphology permanent or transient?

The method was clearly described but only one search strategy was employed, utilising the Medline database. An initial search of the literature often yields further pertinent papers within reference lists, but this approach was not mentioned; nor was the searching of relevant journals by hand which, although time-consuming, may also produce further articles. The Centre for Reviews and Dissemination, at the University of York states that, "Searches for relevant studies can be undertaken initially using electronic databases, but this alone is insufficient" (www. york.ac.uk/inst/crd/report4). Interestingly, this report was cited as being one of the main influences on the search method chosen.

The selection and exclusion criteria were well-described and tabulated. The search revealed 113 articles, of which 100 were excluded. Non-English-language papers were excluded, this group comprising only European papers. The remaining 13 articles were described and the relevant data presented in a digestible tabular format. The control groups and methods were summarised, as well as data on correlations between deformity and prematurity, intubation and sucking habit, and the outcomes and authors' conclusions. A quality analysis was carried out for the 13 studies, of which only three were judged to be of high quality. Because of the lack of homogeneity, no statistical analysis of combined study data could be carried out.

There was some evidence for altered palatal morphology in the short term in premature children, with oral intubation a contributory factor. The results were somewhat contradic- 
tory, however, and the evidence was too weak to conclude whether permanent changes arise. Additionally, some studies specifically excluded digit suckers, which may further confound the results.

All studies demonstrated a delay in dental development and eruption in premature children. When corrected age was applied (ie, when the child would have been born), however, no delay was found. In practice, this period is only up to 3 months; and so is probably of little importance. The effect of prematurity on toothcrown dimensions requires further study. The results from this review demonstrated contradictory findings.

In summary, this is a well-written article about an interesting subject. Any well-conducted systematic review should provide future research directives and this one does indeed make relevant suggestions considering further work in this area. The search strategy was limited and not exhaustive, potentially omitting further relevant papers, but the papers included in this systematic review are clearly presented and analysed.

\section{Practice point}

- Where children present with posterior crossbites or high-vaulted palates, it may be worth asking parents additional questions about prematurity, which could add information related to the aetiology of the malocclusion.

\section{Julian O’Neill}

Kettering General Hospital, Kettering, Northamptonshire, UK

Evidence-Based Dentistry (2005) 6, 41-42.

doi:10.1038/sj.ebd.6400329 